\title{
Expanding the Kidney Donor Pool through Use of Hepatitis C-Infected Donors: is it Time to Dive in?
}

\author{
Gretchen M Kipp ${ }^{1,2}$, Lynsey Biondi ${ }^{1,3}$ and Dinesh Kannabhiran ${ }^{1,4 *}$ \\ ${ }^{1}$ Transplant Alliance, WVU Medicine, USA \\ ${ }^{2}$ Pharmacy and Therapeutics, WVU Medicine, USA \\ ${ }^{3}$ Department of Surgery, WVU Medicine, USA \\ ${ }^{4}$ Department of Medicine, Section of Nephrology, WVU Medicine, USA
}

*Corresponding author: Dinesh Kannabhiran, Transplant Alliance, Department of Medicine, Section of Nephrology, WVU Medicine, Morgantown, WV, USA.

Received Date: September 09, 2019

Published Date: September 13, 2019

\section{Introduction}

The survival benefit of kidney transplantation for patients with end-stage renal disease (ESRD) is well established. However, the demand for kidney donor organs greatly exceeds the current supply. The use of hepatitis $\mathrm{C}$ infected donors could increase the number of kidneys available for transplantation. The use of highly effective second-generation direct acting antivirals (DAAs) has been recently studied for the prevention of chronic hepatitis C virus (HCV) infection in kidney transplant recipients who are HCV negative and receive HCV infected kidney allografts. Small, open-label trials have demonstrated the feasibility of using DAAs as either pre- and post-exposure prophylaxis or as treatment after detection of HCV transmission. Short term outcomes illustrate $100 \%$ prevention of chronic HCV infection with renal function and allograft survival that are comparable to recipients of non-HCV infected kidney donors. Long-term allograft and patient outcomes are required to determine whether the use of HCV infected organs should be considered for all patients with ESRD waiting for kidney transplant. The survival benefit of kidney transplantation for patients with end-stage renal disease (ESRD) is well established [1,2]. However, the demand for kidney donor organs greatly exceeds the current supply which encourages organ procurement organizations and transplant centers to look for innovative strategies to increase the donor pool. Recently, the opioid crisis has increased the number of overdose deaths exponentially [3]. Additionally, the number of hepatitis $\mathrm{C}$ virus (HCV) seropositive donors increased from 452 organs per year to 1506 per year between the years of 2000 and 2016 but only $57 \%$ of the HCV seropositive kidneys were transplanted in 2016 [4]. The use of hepatitis $C$ infected donors could increase the number of kidneys available for transplantation but this strategy has historically been avoided because of risk of HCV transmission as well as inadequate treatment response and risk of rejection with interferon-based regimens. The advent of highly effective secondgeneration direct acting antivirals (DAAs) has increased viral cure of patients with chronic HCV infection to more than 96\% [5-7]. Ongoing research is investigating whether DAAs can be prescribed post-transplant to HCV negative recipients receiving HCV infected donors to increase the donor pool.

The Transplanting Hepatitis C Kidneys Into Negative KidnEy Recipients (THINKER) trial, was a prospective, open-label, nonrandomized trial that included a total of 20 kidney transplant HCV negative recipients who received hepatitis C infected kidneys, determined by HCV nucleic acid testing (NAT) [8,9]. Recipients were included if they were expected to have prolonged times on the wait list while being maintained on dialysis. Recipients could not have any contraindications to liver transplantation or evidence of liver disease detected by FibroScan imaging or serologic testing. Only HCV genotype 1 infected donor kidneys were included. Patients were assessed for HCV transmission on post-op day 3 and were started on elbasvir-grazoprevir (ELB-GRZ) upon detection of HCV infection. Participants with genotype 1 a HCV were tested for baseline nonstructural protein 5A (NS5A) resistance mutations. Patients with genotype $1 \mathrm{a} / 1 \mathrm{~b}$ were treated with ELB-GRZ for a total of 12 weeks while patients with genotype 1a with NS5A resistance mutation were started on ELB-GRZ and ribavirin for 16 weeks total. Outcomes included HCV cure determined by sustained viral response at 12 weeks (SVR12) and adverse events attributable 
to HCV infection or DAA therapy with 1year follow-up. All patients received rabbit antithymocyte globulin induction therapy and triple immunosuppression maintenance with tacrolimus, mycophenolate mofetil, and prednisone. Twenty patients were included in the trial, of which 19 became HCV PCR positive by post-operative day 3 (range 2-4). The last patient became HCV PCR positive on day 5 after transplant. Seventeen donor allografts were infected with HCV genotype 1a and only 3 donor allografts had NS5A mutations. All patients had undetectable HCV PCR within 4 weeks of therapy. Renal function at 12 months was similar between patients who received a hepatitis $C$ infected kidney donor and matched recipients who did not accept hepatitis $\mathrm{C}$ infected kidney donor. No episodes of allograft rejection occurred during treatment. Four patients developed low-level de novo donor specific antibodies (DSAs) that were detected during routine surveillance. Five patients experienced increased but transient elevations in aminotransferase levels which returned to baseline without intervention. One patient with ESRD due to IgA nephropathy was diagnosed with focal segmental glomerulosclerosis post-transplant with no evidence of recurrent IgA nephropathy. Of interest, 50\% of donor organs were excluded in THINKER because of HCV infection that was non-genotype 1 [10]. For this reason, a second trial started, called Exploring Renal Transplants Using Hepatitis C Infected Donors for HCV Negative Recipients (EXPANDER), to utilize these discarded organs [11]. Overall, the methodology of EXPANDER was very similar to THINKER, however, EXPANDER included kidney donor organs infected with genotype 1-4 and DAA therapy was administered as pre- and post-exposure prophylaxis. One dose of ELB-GRZ was administered pre-operatively as pre-exposure prophylaxis. After transplant, patients with genotype $1 \mathrm{a} / 1 \mathrm{~b}$ and 4 were treated with ELB-GRZ for 12 weeks total. Donors with HCV genotype 1a were tested for NS5A mutation and recipients who received a HCV genotype 1a with NS5A mutation kidney allograft were prescribed ELB-GRZ and ribavirin for 16 weeks total. Patients who received genotype 2 or 3 organs were treated with ELB-GRZ and sofosbuvir (SOF) for 12 weeks total. If the donor allograft genotype was unable to be determined due to insufficient viral load, the recipient was treated with ELB-GRZ for 12 weeks.

Ten hepatitis $\mathrm{C}$ negative patients were transplant with hepatitis C infected kidneys. Each patient received one dose of ELB-GRZ prior to transplant. Post-transplant, 7 patients received 12 weeks total of ELB-GRZ for genotype 1,4, or unknown genotypes. No patients received an organ infected with genotype 1a with N5SA mutation. The remaining 3 patients had non-genotype 1 or $4 \mathrm{HCV}$ and were treated with ELB-GRZ and SOF. SVR12 for all patients was $100 \%$. No adverse events occurred related to HCV infection or DAA therapy. Like THINKER, one patient experienced increased aminotransferase levels which increased to 5 times greater than the upper limit of normal (ULN) and then returned to normal without clinical symptoms or intervention. While renal function of HCV infected recipients was not compared to non-HCV infected, matched controls, the median creatinine level at week 12 was $1.05 \mathrm{mg} / \mathrm{dL}$ (IQR: $0.9-2.0 \mathrm{mg} / \mathrm{dL}$ ) and GFR was $63.5 \mathrm{~mL} / \mathrm{min}$ (IQR: 47.8 - 69.9 $\mathrm{mL} / \mathrm{min}$ ). THINKER and EXPANDER both illustrated the feasibility of using highly effective DAA therapy as post-exposure prophylaxis in HCV negative recipients who receive HCV NAT positive donors. The participants of both trials were carefully selected under IRB and the DAA was supplied by pharmaceutical companies. A recent, single center experience by Molnar et al reported outcomes for the use of hepatitis $\mathrm{C}$ infected donors for hepatitis $\mathrm{C}$ negative recipients when used as standard of care treatment [12]. The standard of treatment was offered to all waitlisted recipients regardless of time of the waiting list or dialysis. Allograft donors were considered if they were HCV NAT-positive, donor age of 45 years or younger, and had a donor biopsy with less than $10 \%$ glomerular sclerosis. Recipient HCV PCR was not tested until 4-8 weeks after transplant. Once HCV RNA was positive, HCV genotype was tested and patients were started on glecaprevir-pibrentasvir (GLE/PIB), sofosbuvirvelpatasvir (SOF/VEL), or sofosbuvir/ledipasvir (SOF/LED) for at least 12 weeks as determined by hepatology. The hospital planned to pay for treatment if the third-party payor denied any patient HCV treatment. Standard immunosuppression was rabbit antithymocyte globulin induction with triple immunosuppression of tacrolimus, mycophenolate mofetil, and prednisone maintenance. Between March 1, 2018 and December 31, 2018, the program transplanted 53 hepatitis $\mathrm{C}$ negative kidney transplant recipients with HCV NAT positive donors. All patients had undetectable HCV RNA by 12 weeks of treatment. Renal function was similar to THINKER trial at 6 months post-transplant with eGFR $66 \mathrm{~mL} / \mathrm{min}$ (IQR: 54-79). Forty-seven patients (89\%) were treated with GLE/PIB, 5 patients (9\%) were treated with SOF/VEL, and 1 patient (2\%) was treated with SOF/LED. All insurance plans approved treatment within a median of 5 days of insurance application (IQR: 2-8). Eleven patients originally were denied by the third-party payor but were approved after appeal. Ten patients had AST $>3$ times upper limit of normal and 8 patients had ALT $>3$ times the ULN. All returned to baseline without intervention. One patient developed fibrosing cholestatic hepatitis. This patient was started immediately on DAA therapy and experienced compete resolution. Thirty-two (60\%) of patients experienced low level CMV infection without tissue invasive disease. Eighteen patients (34\%) developed BK viremia and 2 patient developed BK nephropathy on biopsy. Sixteen patients (30\%) developed de novo DSA. Three patients had acute cellular rejection (ACR) and 1 patient had an episode of combined antibody-mediated rejection and ACR.

The short-term outcomes for kidney transplantation of HCV negative recipients using HCV infected kidney donors are excellent. The renal function and allograft survival in clinical trials are comparable to recipients of non-HCV infected kidney donors. Furthermore, the use of HCV infected kidney donors for transplantation decreases the patient's wait time on dialysis [812]. Emerging evidence is addressing some of the initial concerns about the use of HCV infected donors including cost-effectiveness of this strategy $[13,14]$. The study performed Molnar et al further illustrates that third-party insurance companies are paying for the therapy with approval of DAA therapy occurring in less than a week in most cases [12]. 
While initial studies increase the collective understanding of managing this patient population, some questions remain unanswered [8-16]. First, what is the optimal timing of DAA therapy? EXPANDER was the first study to attempt pre- and postexposure DAA prophylaxis strategy [11]. While the number of patients in this study was small, less patients in EXPANDER had elevated transaminase levels compared to either THINKER or the Molnar et al studies leading to the conclusion that avoidance of HCV infection through pre- and post-exposure prophylaxis may help to decrease transaminitis associated with HCV transmission [8-9,11-12]. Moreover, one patient in the Molnar et al. study developed fibrosing cholestatic hepatitis. The authors mention that DAA therapy was started early for this patient and the fibrosing cholestatic hepatitis resolved completely but no additional information was documented. Originally, the treatment protocol for this study started DAA therapy between 4-8 weeks posttransplant but evolved over time to start DAA therapy $<3$ weeks after transplant. Further studies will help to elucidate if pre- and post-exposure DAA therapy is associated with less transaminitis compared to waiting to treat after confirmed HCV transmission. Another interesting finding of the Molnar et al study that is not mentioned in either THINKER or EXPANDER is the development of low level CMV viremia in $60 \%$ of patients and BK viremia in $34 \%$ of patients [8-9,11-12]. Two patients with BK viremia developed biopsy proven BK associated nephropathy (BKAN) but no patients had allograft loss during follow-up. It is unclear whether HCV viremia contributed to the concurrent $\mathrm{CMV}$ and $\mathrm{BK}$ viremias or whether this population of patients were at higher risk of these infections at baseline.

The detection of DSAs was noted in both THINKER and the Molnar et al study [8-9,11-12]. While no rejection episodes occurred during DAA treatment in the THINKER trial, 4 episodes of rejection were documented in the Molnar et al study; 3 episodes of ACR and 1 episode of mixed ACR and antibody mediated rejection. The grades and timing of these rejections were not discussed. It is unclear whether the rejections occurred during or after DAA therapy but it should be noted that the overall incidence of ACR for this cohort is similar to national outcomes [17]. Patients received rabbit antithymocyte globulin for induction therapy with a mean total dose of $4.9 \mathrm{mg} / \mathrm{kg}( \pm 0.9 \mathrm{mg} / \mathrm{kg}$ ). Target tacrolimus goal levels post-transplant and goal mycophenolate mofetil doses were not noted. Future studies should continue to monitor the development of DSAs and rejection in this patient population.

Overall, the utilization of HCV infected kidneys for HCV negative recipients is a promising strategy to increase access to transplantation and should continue to be studied. Current evidence suggests that HCV negative recipients who receive pre- and postexposure DAA prophylaxis or DAA therapy upon detection of HCV transmission can be transplanted with HCV infected kidneys with no documented chronic HCV transmission to date and excellent shortterm renal outcomes. Future studies should investigate the optimal timing of DAA therapy to decrease adverse events described in the literature including the development of DSAs, CMV and BK viremia, and elevated transaminase levels. Long-term allograft and patient outcomes should also be collected to determine whether the use of HCV infected organs should be considered for all patients with ESRD waiting for kidney transplant.

\section{Acknowledgement}

None.

\section{Conflict of Interest}

No conflict of interest.

\section{References}

1. Laupacis A, Keown P, Pus N, Krueger H, Ferguson B, et al. (1996) A study of the quality of life and cost-utility of renal transplantation. Kidney Int 50(1): 235-242

2. Wolfe RA, Ashby VB, Milford EL, Ojo AO, Ettenger RE, et al. (1999) Comparison of mortality in all patients on dialysis, patients on dialysis awaiting transplantation, and recipients of a first cadaveric transplant. $\mathrm{N}$ Engl J Med 341(23): 1725-1730.

3. Durand CM, Bowring MG, Thomas AG, Kucirka LM, Massie AB, et al. (2018) The drug overdose epidemic and decreased-donor transplantation in the United States: a national registry study. Ann Intern Med 168(10): 702-711.

4. Lia AA, Cholaneril G, Cheng XS, Tan JC, Kim D, etal. (2018) Underutilization of hepatitis $C$ virus seropositive donor kidneys in the United States in the current opioid epidemic and direct-acting antiviral era. Diseases 6(3): 62.

5. Zeuzem S, Ghalib R, Reddy KR, Pockros PJ, Ari ZB, et al. (2015) Grazoprevir-Elbasvir Combination Therapy for Treatment-Naive Cirrhotic and Noncirrhotic Patients With Chronic Hepatitis C Virus Genotype 1, 4, or 6 Infection: A Randomized Trial. Ann Intern Med 163(1): 1-13.

6. Kwo PY, Gane EJ, Peng CY, Pearlman B, Vierling JM, et al. (2017) Effectiveness of elbasvir and grazoprevir combination, with or without ribavirin, for treatment-experienced patients with chronic hepatitis $\mathrm{C}$ infection. Gastroenterology 152(1): 164-175.

7. Kwo PY, Poordad F, Asatryan A, Wang S, Wyles DL, et al. (2017) Glecaprevir and pibrentasvir yield high response rates in patients with HCV genotype 1-6 without cirrhosis. J Hepatol 67(2): 263-271.

8. Goldberg DS, Abt PL, Blumberg EA, Van Deerlin VM, Levine M, et al. (2017) Trial of transplantation of HCV-Infected kidneys into uninfected recipients. N Engl J Med 376(24).

9. Reese PP, Abt PL, Blumberg EA, Van Deerlin VM, Bloom RD, et al. (2018) Twelve-month outcomes after transplant of hepatitis C-infected kidneys into uninfected recipients. Annals Intern Med 169(5): 273-281.

10. D Goldberg, V Van Deerlin, M Farooqi, A Sicilia, R Hasz, et al. (2017) Hepatitis $\mathrm{C}$ genotypes among deceased organ donors in the United States [Abstract]. Am J Transplant 17(3): 643.

11. Durand CM, Bowring MG, Brown DM, Chattergoon MA, Massaccesi G, et al. (2018) Direct-acting antiviral prophylaxis in kidney transplantation from hepatitis $\mathrm{C}$ virus-infected donors to non-infected recipients: An open-label nonrandomized trial. Annals Intern Med 168(8): 533-540.

12. Molnar MZ, Nair S, Cseprekal O, Yazawa M, Talwar M, et al. (2019) Transplantation of kidneys from hepatitis C-infected donors to hepatitis C-negative recipients: Single center experience. Am J Transplant.

13. Gupta G, Zhang Y, Carroll NV, Sterling RK (2018) Cost-effectiveness of hepatitis C-positive donor kidney transplantation for hepatitis C-negative recipients with concomitant direct-acting antiviral therapy. Am J Transplant 18(10): 2496-2505.

14. Kadatz M, Klarenbach S, Gill J, Gill JS (2018) Cost-effectiveness of using kidneys from hepatitis $\mathrm{C}$ nucleic acid test-positive donors for transplantation in hepatitis C-negative recipients. Am J Transplant 18(10): 2457-2464. 
15. Friebus Kardash J, Gäckler A, Kribben A, Witzke O, Wedemeyer H, et al. (2019) Successful early sofosbuvir-based antiviral treatment after transplantation of kidneys from HCV-viremic donors into HCV-negative recipients. Transpl Infect Dis.

16. Franco A, Moreso F, Merino E, Sancho A, Kanter J, et al. Renal transplantation from seropositive hepatitis $\mathrm{C}$ virus donors to seronegative recipients in Spain: a prospective study. Transpl Int 32(7): 710-716.

17. Hart A, Smith JM, Skeans MA, Gustafson SK, Wilk AR, et al. (2019) OPTN/ SRTR 2017 Annual Data Report: Kidney. Am J Transplant. 